\title{
FABRICAÇÃO DE CHAPAS DE PARTÍCULAS AGLOMERADAS USANDO GESSO COMO MATERIAL CIMENTANTE
}

\section{MANUFACTURING GYPSUM-BONDED-PARTICLEBOARD WITH RECYCLED PAPER AND PINE WOOD PARTICLES}

\author{
Clovis Roberto Haselein ${ }^{1} \quad$ Leandro Calegari ${ }^{2} \quad$ Luis Fernando Alberti $^{3} \quad$ Adriano Luiz Minello $^{2}$ \\ Paulo Anaximandro da Silva ${ }^{2}$ Rossina Gabriela Figueredo Pintos ${ }^{2}$
}

\begin{abstract}
RESUMO
Neste trabalho, foram confeccionadas chapas aglomeradas, utilizando gesso como material cimentante e papel reciclável dissociado (jornal e offset) e partículas de madeira de pinus como reforços. Em todos os tratamentos, a razão madeira (ou fibras) para gesso foi mantida em 0,25 (base seca) e duas dosagens de água $(\mathrm{w})$ foram empregadas: 0,4 e 0,8 , correspondendo à razão água:gesso. As chapas foram prensadas a frio em prensa de laboratório, em um processo similar à produção de chapas aglomeradas convencionais. Após condicionadas em câmara climatizada, as chapas foram testadas em flexão estática, dureza, arrancamento de parafusos, absorção d'água e inchamento em espessura. Em geral, a adição de fibras causou melhoria nas propriedades das chapas. Diferenças estatísticas significativas em relação à testemunha (gesso puro) foram encontradas para resistência à flexão estática (MOR), dureza e arrancamento de parafusos em relação a alguns dos tratamentos estudados. Já a inclusão de fibras de papel reciclável, com $\mathrm{w}=0,4$, não apresentou diferenças significativas em relação à testemunha para absorção d'água e inchamento em espessura. Os melhores resultados foram encontrados com papel jornal, com um coeficiente $\mathrm{w}=0,4$.
\end{abstract}

Palavras-chave: chapas gesso/madeira, madeira de pinus, papel reciclável.

\begin{abstract}
In this work, gypsum bonded particleboards were manufactured using recycled paper (newspaper and offset) and pine wood particles. In all treatments the ratio wood/gypsum was kept in 0.25 (ovendry base) and two water content $(\mathrm{w})$ were employed: 0.4 and 0.8 , corresponding to the water/gypsum ratio. The boards were cold pressed in laboratory in a process similar to the conventional particlebo ard fabrication. Then, they were tested in static bending, hardness, screw withdrawal and thickness swelling. In general, the addition of fibers improved the board properties. Significant differences were found for some of the treatments for static bending strength, hardness and screw withdrawal, while the inclusion of paper recycled fibers with $\mathrm{w}=0,4$ did not show differences for water absorption and thickness swelling, when compared to conventional gypsum boards. The best results were obtained when newspaper type recyclable fibers were introduced, with $\mathrm{w}=0,4$.
\end{abstract}

Key words: gypsum particleboard, pine wood, recycled paper.

\section{INTRODUÇÃO}

"Gypsum-bonded particleboard (GBP)", ou chapas de partículas liga de gesso, resultam do processo de adição de partículas de madeira a uma matriz de gesso, prensada sob condições ambientais controladas, interagindo com as propriedades físico-mecânicas nas propriedades do produto final.

Os painéis reforçados com liga de gesso para construção têm sido fabricados industrialmente em diversos países já há algum tempo. A primeira planta industrial para fabricação de GBP foi instalada na Finlândia em 1985, e usada desde então em toda a sua capacidade. Apesar de ser relativamente novo no

1. Engenheiro Florestal, PhD., Professor Adjunto do Departamento de Ciências Florestais, Centro de Ciências Rurais, Universidade Federal de Santa Maria, CEP 97105-900, Santa Maria (RS).

2. Acadêmicos do Curso de Graduação em Engenharia Florestal, Centro de Ciências Rurais, Universidade Federal de Santa Maria, CEP 97105-900, Santa Maria (RS).

3. Engenheiro Florestal, Mestrando pelo Programa de Pós-Graduação em Engenharia Florestal Centro de Ciências Rurais, Universidade Federal de Santa Maria, CEP 97105-900, Santa Maria (RS). 
mercado madeireiro, o GBP tem-se difundido popularmente nos anos recentes, particularmente na Europa, em países como Suiça e Alemanha.

As chapas moldadas oferecem muitas vantagens sobre outros conhecidos pain éis para construção existentes no mercado. Possuem boas propriedades de resist ência, consistência uniforme em ambas as faces e boa trabalhabilidade com maquinário normal. Também resistem bem ao fogo (Grexa, 2000), fungos e cupins. Quanto a resistência às variações de umidade, não há consenso. Alguns autores citam as chapas confeccionadas com gesso como sendo pouco resistentes às intempéries e possuindo comportamento muito instável em relação às variações do teor de umidade ambiente (Pasanen et al., 2000), enquanto outros (Gotze et al., 1995) demonstram o contrário.

Por isso, as chapas são indicadas para uso interno, incluindo forros, divisórias e camadas inferiores dos revestimentos dos assoalhos (Hernández-Olivares et al., 1999). Os materiais de reforço, que tem sido usados para melhorar a resistência do gesso, incluem aparas de papel, partículas ou fibras de madeira e esteiras de fibras de vidro.

O presente trabalho foi realizado com o objetivo de testar a qualidade das chapas de gesso reforçadas com partículas de madeira e com papel reciclável (off-set e jornal), usando os processos de fabricação semi-seco e úmido.

\section{REVISÃO BIBLIOGRÁFICA}

Chapas aglomeradas são feitas dos mais variados tipos de materiais, aos quais adiciona-se um adesivo e, por meio de um processo de prensagem das pequenas unidades, forma-se uma peça única. As chapas aglomeradas geralmente são produzidas com resinas sintéticas e os métodos de produção exigem gastos significativos com aquecimento na prensagem e gastos de energia para a secagem. Da mesma forma, a produção dos adesivos orgânicos sintéticos, a maioria derivados de petróleo, geralmente é cara. Já a matéria-prima básica para a formação do GBP são partículas de madeira e gesso, não sendo necessária a prensagem a quente como ocorre com as chapas aglomeradas produzidas com adesivos sint éticos.

Segundo Bauer (1994), o gesso não é um excelente material de colagem, mas pode ser usado como aglutinante da madeira, desde que esta passe por um tratamento com cloreto de cálcio diluído para aumentar a sua capacidade de aderência. Segundo o mesmo autor, quanto mais fino for o gesso melhor serão as suas capacidades adesivas e a sua resistência.

A propriedade que é mais distinta no gesso e que é de grande utilidade para a colagem é a sua possibilidade de ganhar e perder água rapidamente. Com o aquecimento, o gesso perde água, e com o umedecimento, fica com grande plasticidade e moldabilidade. Geralmente, a quantidade de água que possibilita a total re-hidratação do gesso é o quociente w, que é a relação entre quantidade de água e quantidade de gesso. O quociente w, quando assume valores na ordem de 14-21\%, possibilita a re-hidratação do gesso.

No processo de fabricação de aglomerados com gesso, as quantidades usadas de água, superam muito esse valor, e o w varia entre 0,7 - 0,9, significando que existe quase uma parte de água para uma parte de gesso. As pesquisas atuais visam a diminuir o valor de w e também o tempo de endurecimento do aglomerado. A adição de algumas substâncias pode diminuir a quantidade de água usada na fabricação de aglomerados com gesso e madeira.

Tradicionalmente, o GBP é fabricado com o processo úmido no qual o material é misturado com água e formado em esteira. Em anos recentes, esse método convencional foi substituído pelo processo semiseco o qual utiliza menos água (Lempfer et al., 1990). A vantagem do processo semi-seco é que não desperdiça água e, desse modo, requer menos energia para a secagem. A quantidade de água, que é adicionada durante a formação da chapa, fica restrita à quantidade requerida para a reação de hidratação do gesso. As propriedades das chapas de partículas com gesso produzidas no processo semi-seco, são comparáveis ou superiores as de outros produtos de partículas com gesso, como as "plasterboard" e "gypsum-fiberboard".

Mari et al. (1991) trabalhando com fibra de coco (Cocos nucifera L.) e gesso como material 
cimentante, na proporção de 2 ou 3 partes de gesso por parte de fibras e $\mathrm{w}=0,4$ produziram pain éis com qualidade semelhante aos feitos com cimento. Já Duda e Hilbert (1990) mostraram alguns efeitos da dosagem de água na confecção de chapas de gesso/madeira. Segundo estes pesquisadores a chapa possuirá a melhor qualidade quando a água for dosada na mistura das partículas a um nível $w$ de 0,25 . Segundo Lempfer et al. (1990) as chapas aglomeradas produzidas pelo método semi-seco possuem módulo de elasticidade maior que os painéis de gesso/madeira e chapas de pasta (plasterboard) produzidos pelo processo úmido, e não requerem pinturas de proteção ou tratamentos adicionais.

A razão madeira/gesso é de grande importância na confecção das chapas aglomeradas. Para a colagem de fibras de palmeiras a razão madeira/gesso que mostrou melhores resultados foi 1: 3,5 e a densidade ótima foi de $1300 \mathrm{~kg} / \mathrm{m}^{3}$ (Rahim et al., 1991). Uma das vantagens das chapas de partículas aglomeradas produzidas com gesso é a sua resistência ao fogo (Lempfer et al., 1990). A relação entre madeira e gesso, bem como a densidade da chapa, são importantes no que se refere a resistência das chapas ao fogo. Chapas com $25 \%$ de madeira são mais resistentes ao fogo que chapas com $35 \%$ de madeira na su a composição (Dobrowolska e Troger, 1996).

Os extrativos de madeiras, como do álamo (Populus davidiana) e bétula (Betula platyphylla) retardam o endurecimento das chapas produzidas (Zhang, 1990). Segundo Simatupang e Smith (1994), a colagem das chapas aglomeradas feitas com gesso e partículas de madeira de seringueira (Hevea brasiliensis) é retardada por extrativos presentes na madeira, principalmente tanino.

Thole e Weiss (1992) testaram materiais alternativos à madeira na confecção de chapas de partículas aglomeradas e concluíram que o bagaço de cana foi o material mais adequado quando comparado com palha de arroz, bambu, algodão, entre outros. Ainda, segundo estes autores, para a confec ção de chapas com materiais alternativos, deve-se cuidar da participação dos diferentes componentes dos materiais testados, pois alguns não se convertem facilmente em painéis ou chapas, atrapalhando o processo como um todo.

\section{MATERIAL E MÉTODOS}

O presente estudo foi conduzido no Laboratório de Produtos Florestais do Departamento de Ciências Florestais da Universidade Federal de Santa Maria.

As proporções de madeira ou fibras e gesso necessários para a fabricação de cada chapa foram calculados em termos de base seca. Antes da fabricação, determinou-se o teor de umidade de cada um dos componentes (flocos de pinus, papel jornal e papel offset possuíam um valor médio de umidade de 10\%, enquanto que o gesso, $3 \%$ ).

O papel utilizado na fabricação das chapas foi picado a seco em um liquidificador industrial até completa dissociação. As partículas da madeira de Pinus elliottii Engelm foram produzidas em um flaker de laboratório, com dimensões nominais de $20 \times 20 \times 0,6 \mathrm{~mm}$. Para tanto, foram abatidas três árvores retiradas de um povoamento com aproximadamente 15 anos, localizado no Campus da UFSM. Após secagem ao ar, as partículas passaram por peneiras (malhas de $3 \times 3 \mathrm{~mm}$ ) para elimina ção dos finos.

A água foi misturada à madeira ou fibras e esta foi colocada em uma betoneira para ent ão ser adicionado o gesso. Após pronta a mistura dos componentes da chapa, o colchão foi formado em um molde de madeira de 40x40x20 cm, sem fundo, com uma chapa de aço galvanizado colocada nas faces superior e inferior. A espessura final das chapas foi estabelecida em $1 \mathrm{~cm}$ e a massa específica aparente, em 1150 $\mathrm{kg} / \mathrm{m}^{3}$. A razão madeira/gesso foi mantida em todas as chapas em 0,25 (peso seco).

O colchão de partículas e gesso foi prensado em prensa hidráulica marca OMECO, com capacidade para 100 toneladas, durante 30 minutos com uma pressão manométrica de $130 \mathrm{kgf} / \mathrm{cm}^{2}$ (pressão específica de aproximadamente $30 \mathrm{kgf} / \mathrm{cm}^{2}$ ) à temperatura ambiente. Logo após retirada da prensa, a chapa foi levada à câmara climatizada mantida a $20^{\circ} \mathrm{C}$ e $65 \%$ de umidade relativa até peso constante. As chapas foram lixadas levemente e em seguida foram confeccionados os corpos-de-prova. De cada chapa foram retirados dois corpos-de-prova $(28$ x 7,5 cm) para os ensaios de resistência à flexão e dois corpos-de-prova $(15$ x $15 \mathrm{~cm})$ para os testes de inchamento em espessura e absorção d'água. 
Os tratamentos investigados são mostrados na Tabela 1. Para cada tratamento foram feitas três repetições. A proporção de madeira ou fibras para gesso foi de $25 \%$ em todos os tratamentos.

Para determinar o módulo de ruptura (MOR) à flexão, a resistência ao arrancamento de parafusos e a dureza da chapa resultante, foi usada máquina universal de ensaios, hidráulica, marca AMSLER, com capacidade para 20 toneladas. Foi utilizado o material restante, após a realização do teste de flexão, para a realização dos dois últimos ensaios (arrancamento de parafusos e dureza). A avalia ção da absorção d'água e do inchamento em espessura foi realizada após 2 e 24 horas de imersão.

TABELA 1: Tratamentos utilizados na confecção das chapas.

\begin{tabular}{|c|c|}
\hline Tratamentos & Dosagens de água (w) \\
\hline T1 Testemunha - gesso puro & 0,4 \\
\hline T2 Testemunha - gesso puro & 0,8 \\
\hline T3 Gesso mais papel jornal & 0,4 \\
\hline T4 Gesso mais papel offset (sulfite) & 0,4 \\
\hline T5 Gesso mais flocos de pinus & 0,4 \\
\hline T6 Gesso mais papel jornal & 0,8 \\
\hline T7 Gesso mais papel offset (sulfite) & 0,8 \\
\hline T8 Gesso mais flocos de pinus & 0,8 \\
\hline
\end{tabular}

Em que: $w$ = razão água/gesso.

Todos os testes foram realizados segundo a norma ASTM D1037 (1995). Os resultados foram analisados por meio de teste de médias, utilizando o método de Fisher.

\section{RESULTADOS E DISCUSSÃO}

A confecção das chapas aglomeradas, conforme estabelecida para os tratamentos T1 (com gesso puro e coeficiente $\mathrm{w}=0,4$ ) e T8 (gesso mais flocos de pinus e $\mathrm{w}=0,8$ ) não foi possível. No $1^{0}$ caso, observou-se a formação de grânulos de vários tamanhos que não formaram liga suficiente e a conseqüente quebra da chapa ao ser retirada da prensa. A causa mais provável para esse problema pode ter sido a dificuldade de distribuição da água homogeneamente sobre o gesso, já que segundo Bauer (1994), valores de w da ordem de 14 a $21 \%$ possibilitam a completa hidratação do gesso. No $2^{0}$ caso, não ocorreu a cura completa das chapas durante o período estipulado para a prensagem. Possivelmente, se a chapa fosse mantida "grampeada" ou prensada por um período maior do que 30 minutos, a solidificação iria ocorrer. No entanto, esse procedimento foge aos objetivos estipulados para o presente estudo. Por esse motivo, os dois tratamentos foram descartados e apenas o tratamento T2 (com gesso puro e $\mathrm{w}=0,8$ ) foi utilizado como testemunha. Hernández-Olivares et al. (1999) comentam que os valores ideais do quociente w devem estar entre 0,6 e 0,8 . Fora desse intervalo, são normais os problemas de trabalhabilidade. Valores de $w=0,4$, em chapas produzidas com madeira apresentam bons resultados (Fujita et al., 2001), devendo-se, para isso, usar adesivo extra com base em água. No entanto, no pressente estudo foi possível a fabricação de chapas com partículas de madeira e $\mathrm{w}=0,4$.

Todas as demais chapas previstas na Tabela 1 foram confeccionados com sucesso, e os resultados são mostrados nas Tabelas 2 e 3. Mediante a análise de variância dos dados obtidos para a resistência ao arrancamento de parafusos $(\mathrm{F}=6,00$ e $\mathrm{p}=0,43 \%)$, MOR $(\mathrm{F}=3,56$ e $\mathrm{p}=3,02 \%)$ e dureza Janka $(\mathrm{F}=10,48$ e $\mathrm{p}=0,03 \%$ ), pode-se concluir que existem diferenças estatísticas significativas entre os tratamentos a $95 \%$ de probabilidade. A Tabela 2 apresenta a média das propriedades mecânicas e os contrastes para os três testes descritos anteriormente, com relação a cada tratamento.

Em relação ao MOR, os melhores resultados foram encontrados com a inclusão de papel jornal, tanto com coeficiente $\mathrm{w}=0,4$ quanto com coeficiente $\mathrm{w}=0,8$. Já a inclusão de papel offset não causou diferença estatística significativa com relação à testemunha, com nenhum dos coeficientes w utilizados. Por outro lado, o tratamento 7 foi o que apresentou menor valor absoluto de MOR, sendo que os tratamentos -testemunha 4 e 5 não apresentaram diferenças significativas com relação a este. Já para dureza Janka observa-se que o tratamento 3 foi o que apresentou maior valor absoluto, seguido do tratamento 5 o qual n ão diferiu estatisticamente do primeiro. Por outro lado, a testemunha foi a que apresentou menor dureza, $n$ ão 
sendo encontradas diferenças significativas desta em relação ao tratamento 7.

Com exceção da inclusão de papel offset, com $\mathrm{w}=0,8$, todos as demais inclus ões causaram aumento significativo da resistência ao arrancamento de parafusos. Isso também ocorreu para a dureza. Pela Tabela 2, observa-se que o tratamento 5 foi o que apresentou maior resistência ao arrancamento de parafusos; entretanto, não foi estatisticamente diferente dos tratamentos 3, 4 e 6 . Por outro lado, o tratamento -testemunha foi o que apresentou menor resistência ao arrancamento de parafusos, sendo que o tratamento 7 não apresentou diferenças significativas deste.

TABELA 2: Teste de médias para as propriedades mecânicas das chapas coladas com gesso em função dos tratamentos.

\begin{tabular}{|c|c|c|c|c|c|c|c|c|c|c|c|}
\hline \multicolumn{2}{|c|}{ Tratamento } & \multirow{2}{*}{$\begin{array}{l}\text { w } \\
0,8\end{array}$} & \multicolumn{4}{|c|}{$\begin{array}{l}\text { MOR } \\
\text { (MPa) }\end{array}$} & \multicolumn{3}{|c|}{$\begin{array}{c}\text { Dureza Janka } \\
\text { (N) }\end{array}$} & \multicolumn{2}{|c|}{$\begin{array}{c}\text { Arrancamento de } \\
\text { Parafusos } \\
\text { (N) }\end{array}$} \\
\hline $\mathrm{T} 2$ & Testemunha gesso puro & & 2,14 & $\mathrm{a}$ & & & 1049 & $\mathrm{a}$ & & 321 & $\mathrm{a}$ \\
\hline $\mathrm{T} 3$ & Gesso mais papel jornal & 0,4 & 8,35 & & & $\mathrm{c}$ & 6307 & & $C$ & 691 & $b$ \\
\hline $\mathrm{T} 4$ & Gesso mais papel offset & 0,4 & 2,88 & $\mathrm{a}$ & $\mathrm{b}$ & & 4286 & $\mathrm{~b}$ & & 636 & $\mathrm{~b}$ \\
\hline T5 & Gesso mais flocos pinus & 0,4 & 4,13 & $\mathrm{a}$ & $\mathrm{b}$ & & 5012 & $\mathrm{~b}$ & $\mathrm{C}$ & 750 & $\mathrm{~b}$ \\
\hline T6 & Gesso mais papel jornal & 0,8 & 6,06 & & $\mathrm{~b}$ & $\mathrm{c}$ & 4595 & $\mathrm{~b}$ & & 637 & $\mathrm{~b}$ \\
\hline $\mathrm{T} 7$ & Gesso mais papel offset & 0,8 & 0,70 & a & & & 1248 & $\mathrm{a}$ & & 429 & $\mathrm{a}$ \\
\hline
\end{tabular}

Em que: Valores seguidos pela mesma letra, na mesma coluna, não diferem estatisticamente, ao nível de $5 \%$ de probabilidade, pelo teste Least Significant Difference (LSD) de Fisher; w = quociente w (relação entre a quantidade de água e a quantidade de gesso); $\mathrm{T}$ = testemunha; $\mathrm{MOR}=$ módulo de ruptura.

A Tabela 3 apresenta a massa específica aparente, a absorção d'água e o inchamento em espessura após 2 e 24 horas de imersão para cada tratamento. Após 2 horas de imersão em água, não houve diferença estatística significativa entre os tratamentos e entre estes e a testemunha. Pela análise de variância dos dados obtidos da absorção $(\mathrm{F}=5,05 ; \mathrm{p}=0,15 \%$ e cv $=10,86 \%)$ e do inchamento $(\mathrm{F}=23,26 ; \mathrm{p}<0,01 \%$ e cv $=$ $8,54 \%$ ) após 24 horas de imersão em água, pode-se concluir que existem diferenças estatísticas significativas entre esses tratamentos a 95\% de probabilidade. Todos os tratamentos com $\mathrm{w}=0,4$ não diferiram significativamente da testemunha em relação à absorção d'água. Observa-se ainda que a inclusão de flocos de madeira de pinus com um coeficiente $\mathrm{w}=0,4$ (tratamento 5) foi a que apresentou numericamente a menor absorção d'água, apesar desta não diferir estatisticamente da testemunha. Para Okino et al. (1997), no caso da madeira de pinus, a presença da resina pode ocasionar uma maior impermeabilidade das chapas, assim como melhorar as suas características físicas. Felton e De Groot (1996) citam os pinus do Sul dos Estados Unidos como sendo promissores para a fabricação de chapas com materiais cimentantes inorgânicos, uma vez que não apresentam os problemas de liberação de excesso de componentes solúveis em água os quais interferem negativamente na qualidade final da chapa. Com relação ao gesso, segundo os mesmos autores, esse material não apresenta diferenças pronunciadas quanto à espécie utilizada, pois a cura é rápida, sendo maior o número de espécies compatíveis ao processo de fabricação.

Pela Tabela 3, observa-se que o tratamento 4 foi o que apresentou maior inchamento em espessura enquanto que o tratamento 5 não apresentou diferença estatística significativa deste. Já os tratamentos 1 e 2 não diferiram estatisticamente da testemunha. Como os valores encontrados foram muito pequenos (menores do que $1 \%$ ), a precisão de medição tornou-se muito baixa e, por esse motivo, os valores foram omitidos na Tabela 3.

Os valores, tanto de resistência como de inchamento em espessura, encontrados no presente trabalho são similares aos encontrados por Lempfer et al. (1990), e em alguns casos, superiores aos valores obtidos por Hernández-Olivares et al. (1999) para chapas de gesso/cortiça.

O comportamento do inchamento em espessura em conseqüência do quociente $\mathrm{w}$, teor de umidade dos materiais e a relação madeira-fibra/gesso é bastante conhecido. De um modo geral, quando se aumenta a 
razão madeira-fibra/gesso, aumenta-se também a resistência da chapa, diminuindo-se em contrapartida a qualidade desta em relação ao inchamento (Valdez et al., 1989; Mattone, 1990). O primeiro autor cita a razão madeira-fibra/gesso 1:3,5 como sendo ideal para chapas de gesso confeccionadas com baga ço de canade-açúcar ou madeira, obtendo-se resultados ainda melhores, quando se adiciona resina de isocianato (Fujita et al. 2001).

TABELA 3: Teste de médias para as propriedades físicas das chapas em função dos tratamentos.

\begin{tabular}{|c|c|c|c|c|c|c|c|c|c|c|c|c|c|c|}
\hline \multirow{2}{*}{\multicolumn{2}{|c|}{ Tratamento }} & \multirow[t]{2}{*}{ w } & \multirow[t]{2}{*}{$\begin{array}{c}\mathrm{ME} \\
\left(\mathrm{kg} / \mathrm{m}^{3}\right)\end{array}$} & \multicolumn{4}{|c|}{$\begin{array}{c}\text { Absorção d'água } \\
(\%)\end{array}$} & & \multicolumn{6}{|c|}{$\begin{array}{c}\text { Inchamento em Espessura } \\
(\%)\end{array}$} \\
\hline & & & & $\begin{array}{l}2 \mathrm{~h} \\
(\%)\end{array}$ & & $\begin{array}{c}24 \mathrm{~h} \\
(\%)\end{array}$ & & & $\begin{array}{l}2 \mathrm{~h} \\
(\%)\end{array}$ & & & $\begin{array}{l}24 \mathrm{~h} \\
(\%)\end{array}$ & & \\
\hline $\mathrm{T} 2$ & $\begin{array}{l}\text { Testemunha gesso } \\
\text { puro }\end{array}$ & 0,8 & $1100 \mathrm{a}$ & 28,73 & $\mathrm{a}$ & 28,84 & $\mathrm{a}$ & & $<1,00$ & $\mathrm{a}$ & & $<1,00$ & $\mathrm{a}$ & \\
\hline T3 & $\begin{array}{l}\text { Gesso mais papel } \\
\text { jornal }\end{array}$ & 0,4 & $1180 \mathrm{a}$ & 32,05 & $\mathrm{a}$ & 34,20 & $\mathrm{a}$ & & $<1,00$ & $\mathrm{a}$ & & $<1,00$ & $\mathrm{a}$ & \\
\hline $\mathrm{T} 4$ & $\begin{array}{l}\text { Gesso mais papel } \\
\text { offset }\end{array}$ & 0,4 & $1180 \mathrm{a}$ & 33,50 & $\mathrm{a}$ & 33,90 & $\mathrm{a}$ & & $<1,00$ & $\mathrm{a}$ & & $<1,00$ & $\mathrm{a}$ & \\
\hline T5 & $\begin{array}{l}\text { Gesso mais flocos } \\
\text { pinus }\end{array}$ & 0,4 & $1080 \mathrm{a}$ & 27,00 & $\mathrm{a}$ & 28,44 & $\mathrm{a}$ & & 1,55 & $\mathrm{~b}$ & & 1,79 & $\mathrm{~b}$ & \\
\hline T6 & $\begin{array}{l}\text { Gesso mais papel } \\
\text { jornal }\end{array}$ & 0,8 & $1130 \mathrm{a}$ & 36,66 & $\mathrm{a}$ & 43,55 & & b & 4,05 & & $\mathrm{c}$ & 6,83 & & $\mathrm{c}$ \\
\hline $\mathrm{T} 7$ & $\begin{array}{l}\text { Gesso mais papel } \\
\text { offset }\end{array}$ & 0,8 & $1160 \mathrm{a}$ & 34,27 & $\mathrm{a}$ & 36,91 & $\mathrm{a}$ & b & 4,26 & & $\mathrm{c}$ & 5,32 & & $\mathrm{c}$ \\
\hline
\end{tabular}

Em que: Valores seguidos pela mesma letra, na mesma coluna, não diferem estatisticamente, ao nível de 5\% de probabilidade, pelo teste Least Significant Difference (LSD) de Fisher; $w$ = quociente w (relação entre a quantidade de água e a quantidade de gesso); $\mathrm{T}$ = testemunha; $\mathrm{ME}$ =massa específica

As vantagens dos painéis com gesso em relação às chapas tradicionais de madeira aglomerada residem na sua maior estabilidade dimensional, principalmente no que diz respeito ao inchamento em espessura. Já em termos de resistência mecânica, os valores encontrados são menores. Possivelmente, o uso de gessos especiais melhoraria as características de resistência. Outra possibilidade seria a utilização de aditivos como cimento, por exemplo, na quantidade de 10\% (Deng et al., 1998) que permitam um tratamento das partículas de madeira antes da confec ção das chapas, como demonstrado em outros trabalhos (Latorraca, 1996; Zhang, 1990; Teixeira e Guimarães, 1989; Hernández-Olivares et al., 1999; Savastano et al., 2001). Pode-se ainda adicionar uma camada de fibras de papel reciclado na parte externa da chapa, melhorando assim a sua resistência e acabamento, reaproveitando materiais, diminuindo o desperd ício e poluição ambiental (Ince e McKeever, 1994; Felton e De Groot, 1996).

\section{CONCLUSÕES}

A inclusão de fibras de papel jornal em chapas de gesso/madeira causou aumento das propriedades mecânicas das chapas, tais como resistência à flexão estática, dureza e arrancamento de parafusos, em comparação à testemunha.

Foi possível a fabricação de chapas com papel reciclado com inchamento em espessura, após imersão em água por 24 horas, menor do que $1 \%$.

Melhores características das chapas foram encontradas com coeficiente $\mathrm{w}=0,4$, quando comparadas com chapas fabricadas com $\mathrm{w}=0,8$.

\section{REFERÊNCIAS BIBLIOGRÁFICAS}

AMERICAN SOCIETY FOR TESTING AND MATERIALS. ASTM D1037. Evaluating the properties 
of wood-base fiber and particle panel materials. Philadelphia, Pa. 1995.

BAUER, L.A.F. Materiais de construção. 5.ed. Rio de Janeiro: LTC Ed., 1994. v.1.

DENG, Y.H.; FURUNO, T.; UEHARA, T. Improvement on the properties of gypsum particleboard by adding cement. Journal of Wood Science, v. 44, n. 2, p .98-102, 1998.

DOBROWOLSKA, E.; TROGER, F. Fire behavior of gypsum-bonded particleboards. Holz-als-Roh-undWerkstoff, v. 54, n. 3, p .163-169, 1996.

DUDA, A. HILBERT, T. Development of the gypsum matrix structure in gypsum-bonded particleboards. WKI-Mitteilung, n. 511, p. 1-7, 1990.

FELTON, C.C.; DE GROOT, R.C. The recycling potential of preservative-treated wood. Forest Products Journal, v. 46, n. 7/8, p .37-46, 1996.

FUJITA, T; KOMATSU, N.; KAWAI, S. Manufacture and properties of Gypsum-bonded particleboard I. Effect of press time, water-gypsum hemihydrate ratio and resin content on board properties. Mokuzai Gakkaishi, v. 47, n. 2, p .120-128, 2001.

GOTZE, M.; HEMPEL, R.; TAUSCH, K.H. Investigations into the durability of moisture-resistant gypsum wallboard during alternating exposure to heat and moisture. Zement-kalk-gips, v. 48, n. 10, p .555-565, 1995.

GREXA, O. Flame retardant treated wood products. In: WOOD \& FIRE SAFETY, 2000 (partI), Strbské Pleso (Slovak Republic). Anais... Strbské Pleso: Techniká Univerzita vo Zvolene, 2000. p. 101-110

HERNÁNDEZ-OLIVARES, F.; BOLLATI, M.R.; DEL RIO, M.; PARGA-LANDA, B. Development of cork-gypsum composites for building applications. Construction and building materials, n. 13, p.179-186, 1999.

INCE, P.J.; McKEEVER, D.B. Recovery of paper and wood for recycling: Actual and Potential. Madison: United States Department of Agriculture, Forest Service, Forest Products Laboratory. 1994. 11p. (General Techinical Report FPL-GTR-88)

LATORRACA, J.V.F. Estudo da viabilidade do uso da espécie Eucalyptus dunii (Maid) na manufatura de painéis de madeira-cimento. 1996. 94p. Dissertação (Mestrado) - Universidade Federal do Paraná, Curitiba.

LEMPFER, K.; HILBERT, T.; GUNZERODT, H. Development of gypsum particleboard manufacture in Europe. Forests Products Journal, v. 40, n. 6, p. 37-40, 1990.

MARI, E.L. ; PABLO, J.M.V.; PABLO, A.A Production of gypsum-bonded panels from cocowood (Cocos nucifera L.). FPRDI-Journal. v. 20, n. 1-2, p. 73-82, 1991.

MATTONE, R. Comparison between gypsum panels reinforced with vegetable fibers: their behavior in bending and under impact. In: Vegetable plants and their fibbers as buildings. Materials. RILEM, 1990, Salvador. Anais... Salvador: [s.n.], 1990. p. 161-172

OKINO, E.Y.A.; ANDAHUR, M.A.E.S.; SOUZA, M.R. Resistência físico-mecânica de chapas aglomeradas de bagaço de cana-de-açucar modificado quimicamente. Scientia Forestalis, n. 52, p. 35-42, 1997.

RAHIM-S.; KHOZIRAH, S.; KHOZIRAH, S. Effect of wood/gypsum ratio and density on strength properties of gypsum-bonded particleboard from oil palm stems. Journal of Tropical Forest Science, v. 4, n. 1, p. 80-86, 1991.

PASANEN, A.; KASANEN, J.; RAUTIALA, S.; IKAHEIMO, M.; RANTAMAKI, J.; KAARIAINEN, H.; KALLIOKOSKI, P. Fungal growth and survival in building materials under fluctuating moisture and temperature conditions. International Biodeterioration and Biodegradation, n. 46, p. 117-127, 2000.

SAVASTANO Jr., H.; WARDEN, P.G.; COUTTS, R.S.P. Ground iron blast as a matrix for cellulosecement materials. Cement \& Concrete Composites, n. 23, p. 389-397, 2001.

SIMATUPANG, M.A.H.; SCHMITT, U. Wood extractives of rubberwood (Hevea brasiliensis) and their 
influences on the setting of the inorganic binder in gypsum-bonded particleboards. Journal of Tropical Forest Science, v.6, n. 3, p. 269-285, 1994.

TEIXEIRA, D., E.; GUIMARÃES, T., L. Tratamento de partículas de Acácia mearnsii De Wild. para produção de chapas de cimento-madeira. Brasília: Laboratório de Produtos Florestais, IBAMA, 1989. 9p. (Série Técnica, 11)

THOLE, V.; WEISS, D. Suitability of annual plants as additives for gypsum-bonded particleboards. Holz als Roh und Werkstoff, v. 50, n. 6, p. 241-252, 1992.

VALDES, J.L.; PUIG, J.; TORRES, A.; RODRIGUEZ, M.E.; PRADO, R. Bagasso-gypsum boards: preliminary study of the process. Rev. ICDCA, v. 22, n. 3, p.3482, 1989.

ZHANG, Y. Influence of wood extractive on hardening and properties of gypsum plaster and gypsum bonded particleboard. Wood Industry, Beijing, 1990. 